\title{
Self-heating effects in AIGaN/GaN HEMT heterostructures: Electrical and optical characterization
}

\author{
A.V. Naumov, O.F. Kolomys, A.S. Romanyuk, B.I. Tsykaniuk, V.V. Strelchuk, \\ M.P. Trius, A.Yu. Avksentyev, A.E. Belyaev \\ V. Lashkaryov Institute of Semiconductor Physics, NAS of Ukraine, \\ 41, prospect Nauky, 03028 Kyiv, Ukraine; \\ e-mail: naumov_av@ukr.net
}

\begin{abstract}
The effect of self-heating on the transport characteristics and electronic properties of transistor $\mathrm{AlGaN} / \mathrm{GaN}$ heterostructures was investigated. The electrical, micro-Raman and photoluminescence techniques were used for temperature estimations for transistor structures under electrical load. The thermal resistance of these structures has been calculated to obtain the temperature of conducting channel heating from the current-voltage characteristics. The differences in the obtained temperature data from applied techniques have been analyzed.
\end{abstract}

Keywords: self-heating, HEMT, heterostructure, GaN, electronic transport, micro-Raman spectroscopy, photoluminescence.

Manuscript received 23.04.15; revised version received 05.08.15; accepted for publication 28.10.15; published online 03.12.15.

\section{Introduction}

The high electron mobility transistors (HEMT) based on III-nitride $\mathrm{AlGaN} / \mathrm{GaN}$ heterostructures (HS) are promising elements for high-temperature, high-voltage and high-frequency devices of novel micro- and nanoelectronics [1]. These III-nitride HS have great advantages for using in power electronics: high drift electron velocity $\left(v_{s} \sim 3 \cdot 10^{7} \mathrm{~cm} / \mathrm{s}\right)$ under electric fields $E \sim 100 \mathrm{kV} / \mathrm{cm}$, high electric breakdown threshold $\left(\sim 5 \cdot 10^{6} \mathrm{~V} / \mathrm{cm}\right)$, good thermal conductivity $(\sim 1.3 \mathrm{~W} / \mathrm{cm} \mathrm{K})$ and thermal stability (up to $1250 \mathrm{~K}$ ), radiation stability, etc. The essential feature of nitride heterostructures is formation of two-dimensional electron gas (2DEG) [2] with a high density $\left(n_{s} \sim 10^{13} \mathrm{~cm}^{-2}\right)$ localized in quantum well at GaNAlGaN interface. This effect occurs due to high $\left(\sim 10^{5} \mathrm{~V} / \mathrm{cm}\right)$ spontaneous and piezo-polarization fields that exist in such III-nitride heterostructures.

However, when III-nitride transistors operate at the high electrical load $(>10 \mathrm{~W} / \mathrm{mm})$ the negative factor appears - Joule self-heating in the conducting channel with 2DEG. This factor limits the efficiency of transistor devices: self-heating leads to degradation of device electrical parameters and to appearance of heat removal problem [3]. Therefore, exact determination of temperature in conductive part of HEMT structures is important for optimization of design and operation modes of these structures.

The broad spectrum of experimental and theoretical methods were used for temperature estimations in 2DEG conducting channel and heterostructures at whole: electrical DC and pulse measurements [4-6], micro-Raman and infrared (IR) 
thermography $[7,8]$, numerical calculations and modeling $[9,10]$. Also, complex investigations of selfheating in HEMT structures were made to compare electrical and optical temperature data. In the study by N. Killat et al. [11], the relative accuracy of HEMT heating temperature estimations was tested using Raman spectroscopy, IR thermography and electrical measurements. The authors used micro-Raman technique to control the results of heat-conduction processes modeling. It was shown that pulse currentvoltage measurements give the temperature values of conducting 2DEG channel heating by $7 \%$ lower than Raman and modeling data, and IR thermography gives unrealistic $45 \%$ lower temperature values. In the earlier work of these authors [12], two electrical DC methods of self-heating temperature determination in HEMT were compared, and Raman spectroscopy was used as a reference method. It was shown that electrical DC methods give considerable underestimation of the temperature $(>50 \%)$. However, all the above mentioned experimental techniques give different temperature estimations for each specified HEMT structures, and there is no general consensus in the interpretation of obtained results. The key point here is to understand the basic physical processes to which all the stated methods of assessment of temperature are sensitive.

The objective of this work was to make complex characterization of $\mathrm{AlGaN} / \mathrm{GaN}$ HEMT-structures by electrical, micro-Raman and photoluminescence (PL) measurements under applied electrical fields, to compare temperature estimations from different methods and to ascertain the relationship between the optical and electrical characteristics.

\section{Samples}

The AlGaN/GaN HEMT heterostructures used in this work were grown by metal-organic chemical vapor deposition on a sapphire substrate $(450 \mu \mathrm{m})$. HS consisted of $\mathrm{GaN}$ undoped buffer layer $(3 \mu \mathrm{m})$, $\mathrm{Al}_{0.3} \mathrm{Ga}_{0.7} \mathrm{~N}$ barrier layer $(30 \mathrm{~nm})$ and $\mathrm{GaN}$ undoped cap layer $(4 \ldots .5 \mathrm{~nm}$ ) (inset in Fig. 1a). The samples include transmission line model (TLM) patterns with sections of $160 \mu \mathrm{m}$ width and different length $(30 \ldots 180 \mu \mathrm{m})$ of conducting channel and Hall-bar pattern for 2DEG characterization formed by photolithography on the AlGaN/GaN HS (Fig. 1b). Ohmic contacts were fabricated by deposition of $\mathrm{Ti} / \mathrm{Al} / \mathrm{Ni} / \mathrm{Au}$ metallization annealed for $40 \mathrm{~s}$ at $800^{\circ} \mathrm{C}$.

The self-consisted calculations of energy bands structure and electronic states density for such AlGaN/GaN HEMT structures indicated that 2DEG is localized within 2 to $4 \mathrm{~nm}$ layer in an asymmetrical quantum well $(\mathrm{QW})$ at the $\mathrm{GaN}-\mathrm{AlGaN}$ heterointerface (Fig. 1a). Transport characteristics of 2DEG in the conducting channel were obtained using Hall measurements: electron mobility $\mu_{300 \mathrm{~K}}=1260 \mathrm{~cm}^{2} / \mathrm{V} \cdot \mathrm{s}$, 2DEG concentration $n_{S}=1.02 \cdot 10^{13} \mathrm{~cm}^{-2}$.

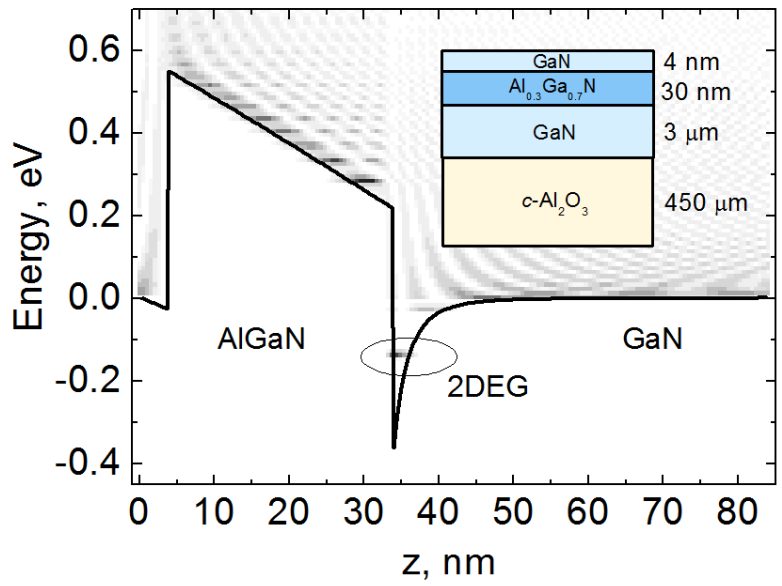

(a)

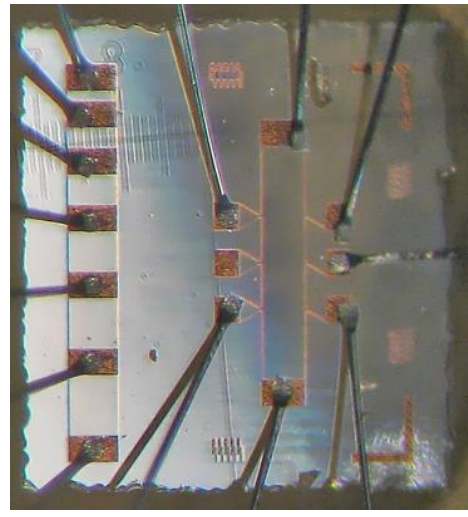

(b)

Fig. 1. (a) Schematic sample structure, calculated energy band profile and electronic states density; (b) View of experimental $\mathrm{Al}_{0.3} \mathrm{Ga}_{0.7} \mathrm{~N} / \mathrm{GaN}$ HEMT sample with TLM and Hall-bar patterns.

\section{Electrical measurements}

Current-voltage characteristics (CVCs) of $\mathrm{Al}_{0.3} \mathrm{Ga}_{0.7} \mathrm{~N} / \mathrm{GaN}$ HEMT sample were obtained using source-measure unit Keithley model 2601 in the DC regime. The sample was placed on a copper heat sink plate in the electro-optical cryostat. Fig. 2 shows typical CVCs for the conducting channel lengths of 30,90 and $180 \mu \mathrm{m}$, measured at $300 \mathrm{~K}$.

All CVCs curves show linear behavior at low voltages up to $\sim 1.5 \mathrm{~V}$. Next, they become sub-linear and at high voltages $>10 \mathrm{~V}$ CVCs have saturation region. In addition, short channel with $L=30 \mu \mathrm{m}$ reveals region of negative different conductance (NDC) after $15 \mathrm{~V}$ due to the self-heating effects $[13,14]$. Fig. $2 b$ illustrates the dependence of current on electric filed, $I(E)$. Additionally, from linear ohmic parts of CVCs measured on TLM patterns, we calculated the contact resistance $R_{c} \sim 1.5 \mathrm{Ohm}$. 


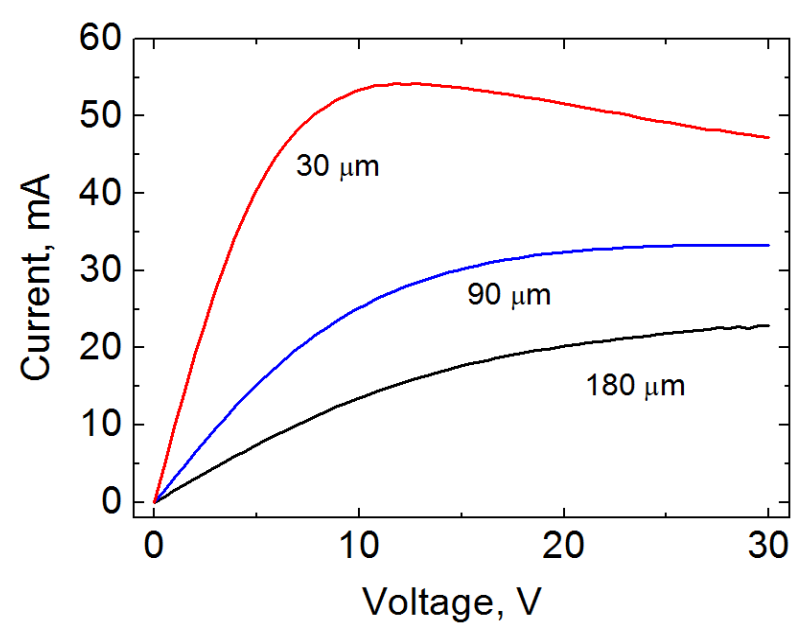

(a)

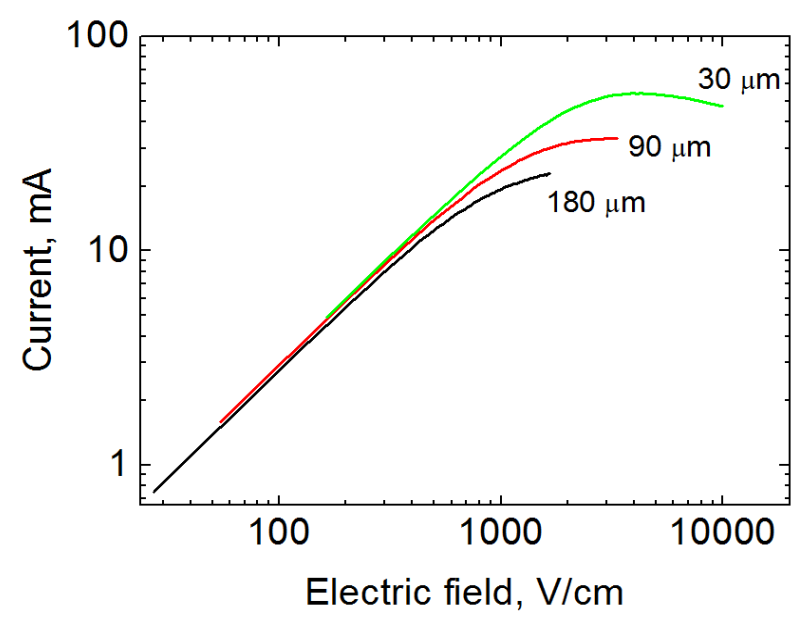

(b)

Fig. 2. (a) Current-voltage characteristics for different length of conducting channel $(30,90,180 \mu \mathrm{m})$ measured on the TLMstructure; (b) The same characteristics in the $I(E)$ format. $T=300 \mathrm{~K}$.

\section{Theoretical calculations of thermal conductivity}

To obtain the temperature of conducting channel heating, we made calculations of thermal resistance of $\mathrm{Al}_{0.3} \mathrm{Ga}_{0.7} \mathrm{~N} / \mathrm{GaN}$ HEMT structure on the base of simplified model of heat diffusion and dissipation [4] (Fig. 3). It was assumed that the thermal resistance was mainly determined by the sapphire substrate, because the buffer layer and active region of the heterostructure (conducting channel) have two orders of magnitude smaller thickness and several times higher thermal conductivity $\left(\lambda_{\mathrm{GaN}} \sim 130 \mathrm{~W} /(\mathrm{m} \cdot \mathrm{K})\right)$ as compared to sapphire $\left(\lambda_{\mathrm{Al} 2 \mathrm{O} 3} \sim 30 \mathrm{~W} /(\mathrm{m} \cdot \mathrm{K})\right) \quad[12]$. Also, for simplicity, we neglect the temperature dependence of the thermal conductivity of the sapphire substrate, $\lambda(T)$. In this case, the problem for modeling of heat transfer is reduced to the Laplace equations [15].

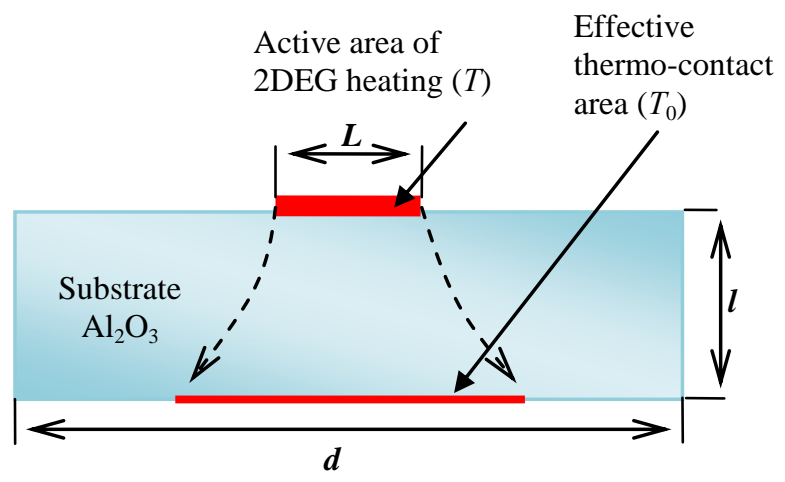

Fig. 3. Schematic model of heat exchange processes in the HEMT heterostructure. The active area is defined as a part of the 2DEG channel between two TLM contacts.

On the one hand, the thermal resistance for the constant cross-section along heat flux can be written as $\Theta=l /\left(\lambda \cdot S_{\text {eff }}\right)$, where $S_{\text {eff }}$ is the effective area of thermal contact, $\lambda$ - coefficient of thermal conductivity, $l-$ thickness of the structure. On the other hand, the thermal resistance is associated with heat dissipation during flow of current and, therefore, with the dissipated power ( $\left.P_{\text {dis }}=I \cdot E \cdot L\right)$, and the temperature rise $\Delta T$ in the conducting channel as $\Theta=\Delta T / P_{d i s}$. Here, $L$ is the length of the conductive channel, $I$ - current in the channel, $E=V / L-$ average electric field in the channel, $\Delta T=T-T_{0} \quad(T-$ temperature of the active region (heating of $2 \mathrm{DEG}) ; T_{0}$ - temperature of the effective thermal contact).

In the linear approximation we can write: the effective thermal contact area $S_{\text {eff }}$ is defined as $S_{\text {eff }}=d^{2} /[1+\sigma(L / d, h / d, l / d)]$. Here, the function $\sigma=2\left(\sigma_{1}+2 \sigma_{2}\right)$ is defined by infinite series:

$\sigma_{1}=\sum_{n}\left[\varphi_{n}^{2}(h / d)+\varphi_{n}^{2}(L / d)\right] \psi_{n=m}(l / d \sqrt{2})$,

$\sigma_{2}=\sum_{n, m=1}^{\infty} \varphi_{n}^{2}(L / d) \varphi_{m}^{2}(h / d) \psi_{n, m}(l / d)$,

where $\varphi_{n}(x)=\sin (n \pi x) / n \pi x$,

$\psi_{n, m}(x)=\tanh \left(2 \pi x \sqrt{n^{2}+m^{2}}\right) /\left(2 \pi x \sqrt{n^{2}+m^{2}}\right)$.

Parameters for the calculation of thermal resistance: thickness of the heterostructure $l=450 \mu \mathrm{m}$ (the sapphire substrate); the channel length $L=30 / 90 / 180 \mu \mathrm{m}$; the channel width $h=160 \mu \mathrm{m}$; substrate width $d=1.5 \mathrm{~mm}$. We obtained the following values of thermal resistance for different channel lengths: $\Theta_{30 \mu \mathrm{m}}=186 \mathrm{~K} / \mathrm{W}$, $\Theta_{90 \mu \mathrm{m}}=120 \mathrm{~K} / \mathrm{W}, \Theta_{180 \mu \mathrm{m}}=85 \mathrm{~K} / \mathrm{W}$. Using the data obtained from the experimental CVCs, we calculated the dependence of the temperature of active region on the applied voltage for different lengths of the conducting channel (Fig. 7). 


\section{Optical measurements}

The second stage of this work involved micro-Raman spectroscopy to the direct monitoring of the samples temperature in the operation regime. This non-destructive non-invasive method, based on the known relationship between the phonon frequency and the temperature, is an effective way to measure the temperature of objects [16]. Measurements of the Raman spectrum on a $180 \mu \mathrm{m}$ channel of TLM-structure were made by microspectrometer JYT-64000 with excitation at $325 \mathrm{~nm}$ in the backscattering mode. Fig. 4 shows a typical Raman spectrum of $\mathrm{Al}_{0.3} \mathrm{Ga}_{0.7} \mathrm{~N} / \mathrm{GaN}$ HEMT-structure measured in the equilibrium state without applied electrical field at $300 \mathrm{~K}$. The focusing was made in the middle of the heterostructure (in Fig. 4, phonon peaks of GaN layer and sapphire substrate are presented).

The temperature of the sample in the operation mode, during application of electric fields, was determined, firstly, from the ratio of the integral intensities of antiStokes and Stokes phonon lines of Raman spectrum:

$\frac{I_{S}}{I_{A S}} \cong C\left(\frac{\omega_{l}+\omega_{S}(T)}{\omega_{l}-\omega_{S}(T)}\right)^{4} \cdot e^{\frac{\hbar \omega_{S}(T)}{k T}}$

here $\omega_{l}, \omega_{S}$ are the frequencies of the exciting laser light and the Stokes component of the phonon mode $E_{2}^{\text {high }}$ respectively. The calibration factor $C$ depends on the experimental conditions and is determined by the efficiency of light scattering and the registration conditions of Raman spectra in the Stokes and antiStokes regions (depending on the absorption coefficient, the light scattering cross section, the resonance conditions, the spectral sensitivity of the spectrometer). Determination of the temperature from this relationship $\left(I_{s} / I_{a s}\right)$ becomes possible due to strong dependence of the anti-Stokes line on the temperature.

It should be mentioned that in equation (1), the temperature $T$ is the phonon temperature. Accordingly, if there are non-equilibrium hot phonons, the temperature obtained from the ratio $I_{s} / I_{a s}$, will be higher than the actual lattice temperature. In the absence of hot phonons the temperature $T$ corresponds to the lattice temperature.

Secondly, the temperature of the sample was obtained from the shift of $E_{2}{ }^{\text {high }}$ phonon mode, which can be analytically represented as:

$$
\omega(T)=\omega_{0}-\frac{A}{e^{B\left(\frac{h c \omega_{0}}{k T}\right)}-1} .
$$

The $E_{2}^{\text {high }}$ phonon mode peak is shifted to lower frequencies when electric field is applied and the sample is heated (Fig. 5). The approximating parameters $A, B$, and $C$ in this study were determined using the temperature calibration of Raman spectra by controlled heating of the sample in a cryostat without application of the electric field.

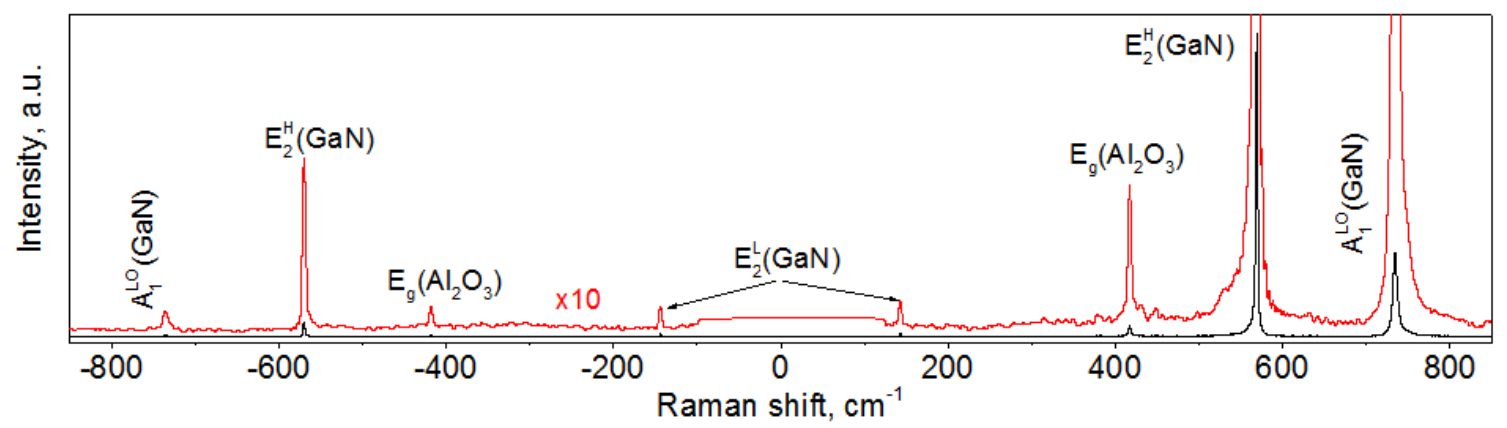

Fig. 4. Typical Raman spectrum of HEMT-structure $\mathrm{Al}_{0.3} \mathrm{Ga}_{0.7} \mathrm{~N} / \mathrm{GaN}$. $T=300 \mathrm{~K}$, the channel length $L=180 \mu \mathrm{m}$.
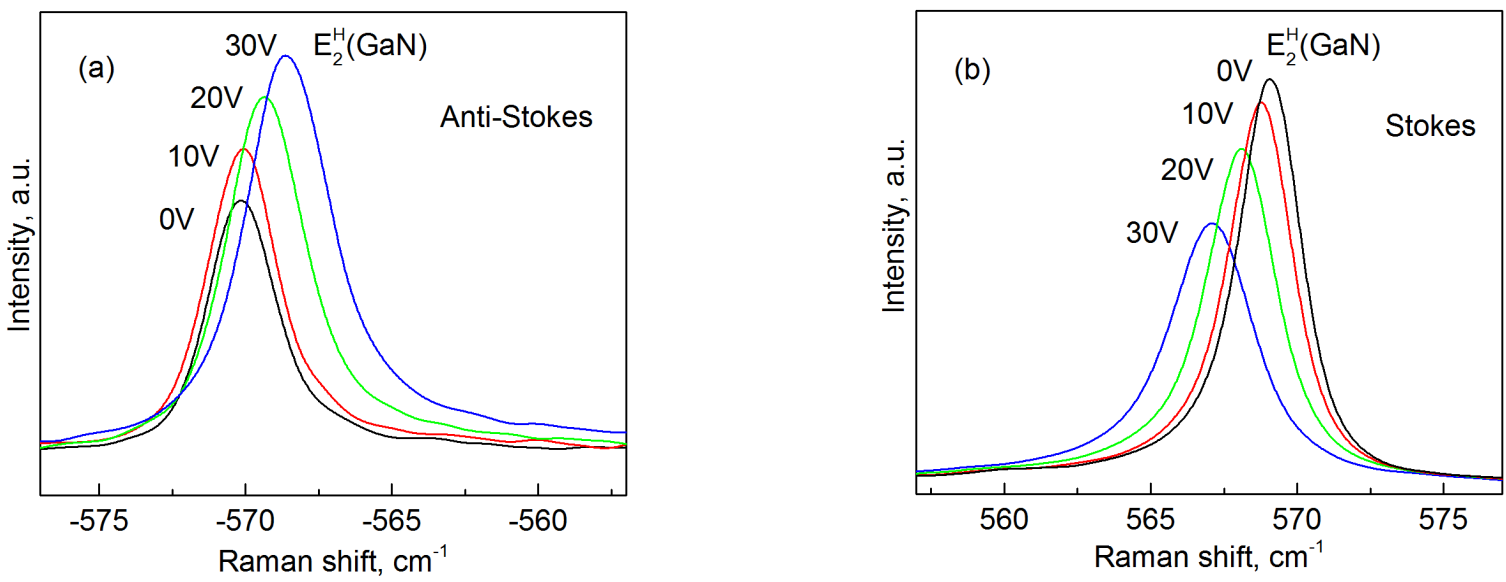

Fig. 5. Anti-Stokes (a) and Stokes (b) Raman spectra of E2high $(\mathrm{GaN})$ mode of the HEMT-structure Al0.3Ga0.7N/GaN. The channel length $\mathrm{L}=180 \mu \mathrm{m}$. 
In addition to the Raman investigations, the edge photoluminescence spectra were obtained for samples under an electrical load (Fig. 6a). We registered a shift of the PL peak into the red region of the spectrum, which is also determined by heating of the sample [17]. In order to associate the value of PL peak shift with a temperature of heating, we also made direct calibration PL spectra were measured without applied voltage, using the passive heating of the sample in the thermostat.

The obtained values of edge PL peak shift (Fig. 6b) were approximately described by the Varshni formula [18] for the temperature dependence of the semiconductor band gap $E_{g}=E_{0}-\alpha T^{2} /(T+\beta)$. Here, $\alpha$ and $\beta$ are Varshni fitting coefficients, $E_{0}$ is the energy transition at $0 \mathrm{~K}$. Our fitting parameters were: $\alpha=7.59 \cdot 10^{-4}, \beta=1000, E_{0}=3.475 \mathrm{eV}$.

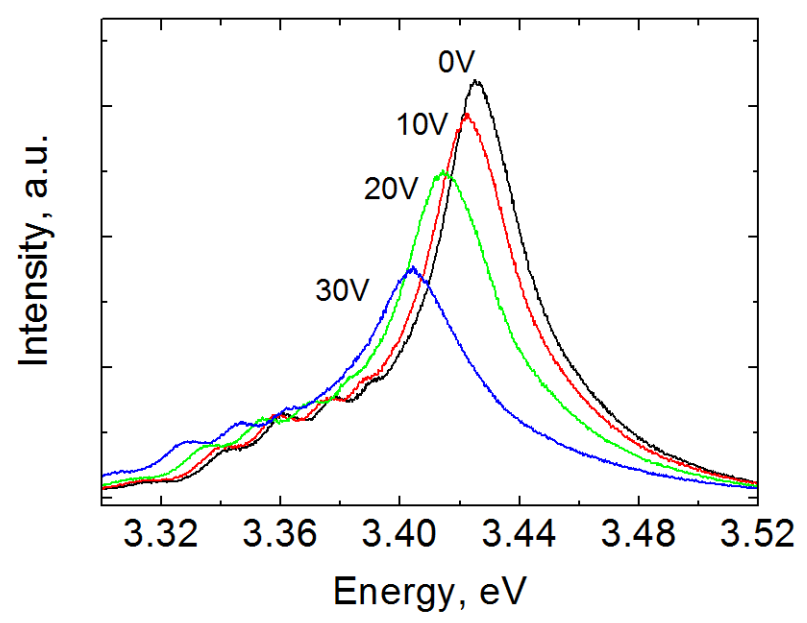

(a)

\section{Discussion}

Fig. 7a, b shows a summary data on the heating temperature for most representative $30 \mu \mathrm{m}$ and $180 \mu \mathrm{m}$ lengths of TLM channels in HEMT-structure. The points in this graph relate to optical measurements at typical voltages of 5,10,20 and $30 \mathrm{~V}$ and corresponding values of the electrical field. The temperature curves obtained using Raman measurements show the highest temperature values. The results based on electrical measurements in comparison with the Raman measurements give temperature values by $20 \ldots 30$ degrees and 150 degrees less for $180-\mu \mathrm{m}$ and $30-\mu \mathrm{m}$ TLM-channels, respectively. The photoluminescence results are very close to the electrical data and not shown in the figure.

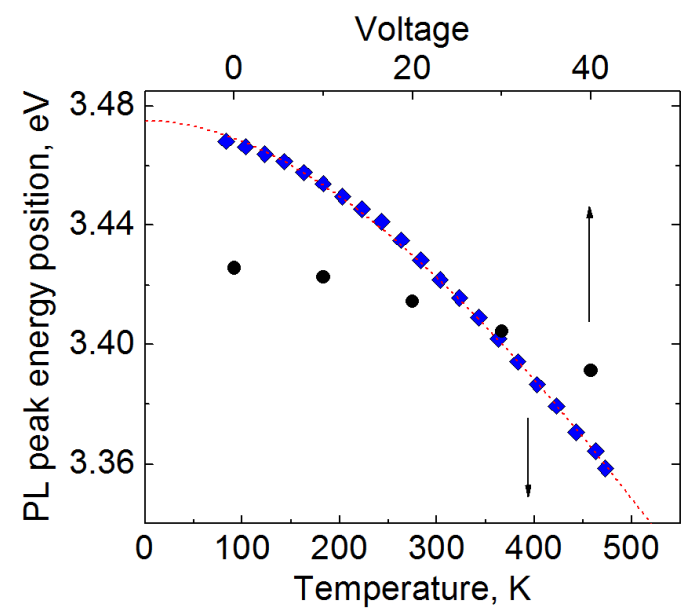

(b)

Fig. 6. (a) PL spectra of the HEMT structure $\mathrm{Al}_{0.3} \mathrm{Ga}_{0.7} \mathrm{~N} / \mathrm{GaN}$ under applied voltages up to $30 \mathrm{~V}$. $T=300 \mathrm{~K}$, the channel length $L=$ $180 \mu \mathrm{m}$. (b) The calibration dependence of edge PL peak position on the sample temperature in thermostat (squares); the approximation based on the Varshni formula (red line); dependence of edge PL peak position on the voltage applied to the sample (circles).

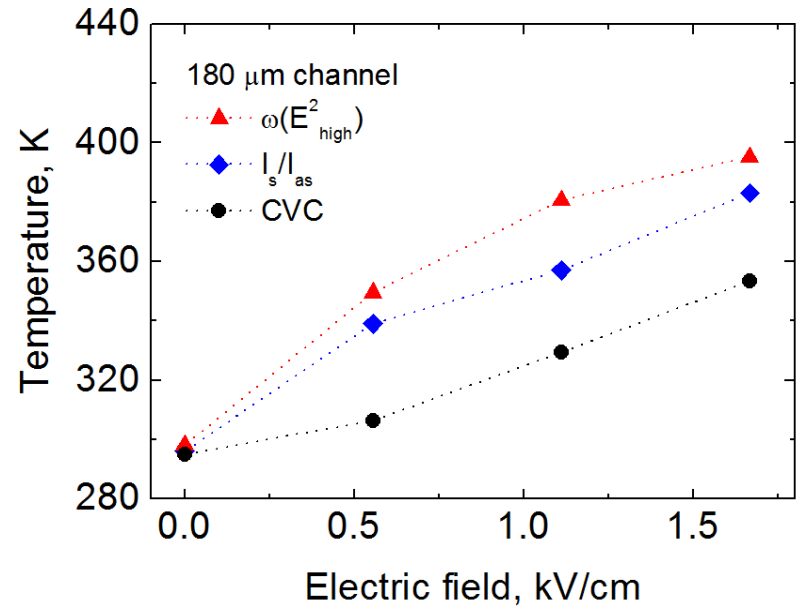

(a)

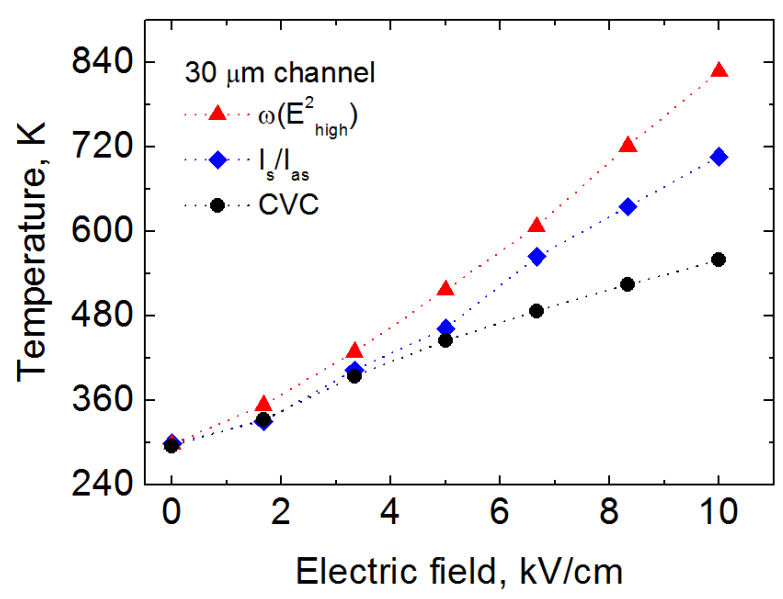

(b)

Fig. 7. Summary data of heating temperature for 30 (a) and $180 \mu \mathrm{m}$ (b) TLM-channels in the HEMT-structure $\mathrm{Al}_{0.3} \mathrm{Ga}_{0.7} \mathrm{~N} / \mathrm{GaN}$ obtained using optical and electrical methods. 
We used two approaches to determine the temperature of the HEMT structure by micro-Raman measurements - by the frequency shift of the phonon mode $\left(E_{2}{ }^{\text {high }}\right)$ and by the ratio of the intensities of phonon mode $\left(E_{2}{ }^{\text {high }}\right)$ in Stokes and anti-Stokes spectra. The temperature values determined by these two approaches for various applied electric fields differ only by $5 \ldots 10 \mathrm{~K}$ for $180-\mu \mathrm{m}$ and about $40 \ldots 120 \mathrm{~K}$ for $30-\mu \mathrm{m}$ channels. The frequency shift $\Delta \omega\left(E_{2}{ }^{\text {high }}\right)$ corresponds to the temperature of the lattice, whereas the ratio $I_{s} / I_{a s}$ is responsible for the temperature of the phonon population and sensitive to the appearance of non-equilibrium (hot) phonons. Since the temperature dependences are almost the same for $180-\mu \mathrm{m}$ TLMchannel, probably hot phonons are absent, and hot electrons are absent, too (hot electrons produce an additional population of non-equilibrium phonons with temperature higher than the temperature of the lattice). Indeed, electric fields in a long channel with $L=180 \mu \mathrm{m}$ are too small for the occurrence of hot electrons (up to $\sim 1.6 \mathrm{kV} / \mathrm{cm}$ at $30 \mathrm{~V}$ ). For $30-\mu \mathrm{m}$ channel, the difference in temperature values obtained from the $E_{2}{ }^{\text {high }}$ position shift and the Stokes/antiStokes ratio becomes more significant at high electric fields $(10 \mathrm{kV} / \mathrm{cm}$ at $30 \mathrm{~V})$, and $E_{2}^{\text {high }}$ curve is also above the $I_{s} / I_{a s}$ curve. This difference may be related to additional elastic deformation contribution to $E_{2}^{\text {high }}$ position shift during thermal expansion of the lattice at high temperatures [19].

Photoluminescence measurements (Fig. 6) also clearly show the presence of heating of the sample. The results based on electrical measurements and photoluminescence spectra both are in good agreement, but in comparison with the Raman spectra they are understated. It is because both PL and CVCs are responsible for the entire volume of the sample and give the average temperature of the whole sample. While the micro-Raman technique has a high spatial resolution $(\sim 0.7 \mu \mathrm{m})$ and when we focus at a certain point of TLMsection of the sample, we cover a very small region of the sample and get the temperature of local overheated region of the conductive 2DEG-channel.

\section{Conclusions}

The effects of Joule self-heating in $\mathrm{Al}_{0.3} \mathrm{Ga}_{0.7} \mathrm{~N} / \mathrm{GaN}$ HEMT-structures under applied electrical fields were investigated using a complex of optical and electrical diagnostics methods. Estimations of the temperature values as a function of applied voltage have been made. The observed differences between the temperature data obtained using different methods, in particular high temperatures obtained by micro-Raman measurements, are related to local overheating of 2DEG conducting channel, which gives the main contribution to the Raman spectra, while the photoluminescence and electrical measurements characterize the average temperature of entire heterostructure sample. The results will be useful in developing new devices based on $\mathrm{AlGaN} / \mathrm{GaN}$ structures for high temperature, high voltage and highfrequency electronics of new generation.

\section{References}

1. D.J. Cheney, E.A. Douglas, L. Liu et al., Reliability studies of $\mathrm{AlGaN} / \mathrm{GaN}$ high electron mobility transistors // Semicond. Sci. Technol. 28, 074019 (2013).

2. J.P. Ibbetson, P.T. Fini, K.D. Ness, S.P. DenBaars, J.S. Speck, U.K. Mishra, Polarization effects, surface states, and the source of electrons in AlGaN/GaN heterostructure field effect transistors // Appl. Phys. Lett. 77(2), p. 250 (2000).

3. Z. Yan, G. Liu, J.M. Khan, A.A. Balandin, Graphene quilts for thermal management of highpower GaN transistors // Nature Communs. 3, P. 827 (2012).

4. S.P. McAlister, J.A. Bardwell, S. Haffouz, H. Tang, Self-heating and the temperature dependence of the dc characteristics of $\mathrm{GaN}$ heterostructure field effect transistors // J. Vac. Sci. Technol. A, 24(3), p. 624 (2006).

5. S.A. Vitusevich, S.V. Danylyuk, N. Klein et al., Separation of hot-electron and self-heating effects in two-dimensional AlGaN/GaN-based conducting channels // Appl. Phys. Lett. 82(5), p. 748 (2003).

6. J. Kuzmik, P. Javorka, A. Alam, M. Marso, M. Heuken, P. Kordos, Determination of channel temperature in $\mathrm{AlGaN} / \mathrm{GaN}$ HEMTs grown on sapphire and silicon substrates using DC characterization method // IEEE Trans. Electron Devices, 49(8), p. 1496 (2002).

7. S. Choi, E.R. Heller, D. Dorsey, R. Vetury, S. Graham, Thermometry of AlGaN/GaN HEMTs using multispectral Raman features // IEEE Trans. Electron Devices, 60(6), p. 1898-1904 (2013).

8. A. Sarua, H. Ji, M. Kuball, M.J. Uren, T. Martin, K.P. Hilton, R.S. Balmer, Integrated microRaman/infrared thermography probe for monitoring of self-heating in $\mathrm{AlGaN} / \mathrm{GaN}$ transistor structures // IEEE Trans. Electron Devices, 53(10), p. 2438 (2006).

9. Y. Zhang, S. Feng ,H. Zhu, J. Zhang, B. Deng, Two-dimensional transient simulations of the selfheating effects in GaN-based HEMTs // Microelectronics Reliability, 53, P.694-700 (2013).

10. S. Vitanov, V. Palankovski, S. Maroldt, R. Quay, High-temperature modeling of $\mathrm{AlGaN} / \mathrm{GaN}$ HEMTs // Solid-State Electron. 54, p. 1105 (2010).

11. N. Killat, M. Kuball, T.-M. Chou, U. Chowdhury, J. Jimenez, Temperature assessment of AlGaN/GaN HEMTs: A comparative study by Raman, electrical and IR thermography // International Reliability Physics Symposium, p. 528 (2010). 
12. R.J.T. Simms, J.W. Pomeroy, M.J. Uren, T. Martin, M. Kuball, Channel temperature determination in high-power AlGaN/GaN HFETs using electrical methods and Raman spectroscopy // IEEE Trans. Electron Devices, 55(2), p. 478 (2008).

13. M. Gonschorek, J.-F. Carlin, E. Feltin, M.A. Py, N. Grandjean, Self-heating in AlInN/AlN/GaN high power devices: Origin and impact on contact breakdown and IV characteristics // J. Appl. Phys. 109, 063720 (2011).

14. W.D. Hu, X.S. Chen, Z.J. Quan, C.S. Xia, W. Lub, P.D. Ye, Self-heating simulation of GaN-based metal-oxide-semiconductor high-electron-mobility transistors including hot electron and quantum effects // J. Appl. Phys. 100, 074501 (2006),

15. R. Bellman (Ed.), Mathematics in Science and Engineering. Academic Press, New York, 1965.
16. M. Kuball, J.M. Hayes, M.J. Uren, T. Martin, J.C.H. Birbeck, R.S. Balmer, B.T. Hughes, Measurement of temperature in active high-power AlGaN/GaN HFETs using Raman spectroscopy // IEEE Electron Device Lett. 23(7), p. 7 (2002).

17. B. Monemar, Fundamental energy gap of GaN from photoluminescence excitation spectra // Phys. Rev. B, 10(2), p. 676 (1974).

18. Y.P. Varshni, Temperature dependence of the energy gap in semiconductors // Physica, 34, p. 149-154 (1967).

19. A. Link, K. Bitzer, W. Limmer et al., Temperature dependence of the $\mathrm{E}_{2}$ and $\mathrm{A}_{1}(\mathrm{LO})$ phonons in $\mathrm{GaN}$ and AlN // J. Appl. Phys. 86(11), p. 6256 (1999). 